\title{
Bio-Soliton Condensation in Human Body
}

\author{
Qiao Bi \\ Department of Physics, Science School, Wuhan University of Technology, Wuhan, China \\ Email:biqiao@gmail.com
}

How to cite this paper: Bi, Q. (2017) BioSoliton Condensation in Human Body. Journal of Modern Physics, 8, 315-322. https://doi.org/10.4236/jmp.2017.83020

Received: January 3, 2017

Accepted: February 25, 2017

Published: February 28, 2017

Copyright $\odot 2017$ by author and Scientific Research Publishing Inc. This work is licensed under the Creative Commons Attribution International License (CC BY 4.0).

http://creativecommons.org/licenses/by/4.0/

\begin{abstract}
In this work, we try to build a model to describe the condensation through the couple of bio-solitons in a well based on the BCS theory extended by the nonlinear operator, which can be used to simulate the created process of the internal elixir in the middle of the abdomen of the human body. The goal of this work is to provide a mechanism to explain the effect of stored bio-energy in the bio-systems.
\end{abstract}

\section{Keywords}

Bio-Soliton, Condensation, Nonlinear Oscillation

\section{Introduction}

The Davydov soliton is a fundamental nonlinear excitation in the bio-system of human being, which has been discovered by the Davydov in 1973 [1] [2] [3]. By improving his model, Pan Xiao-feng presented that the Davydov soliton is the basic transformed unit of energy and information in bio-system included human body [4] [5]. Many facts also reveal that the bio-solitons sometimes can be seen as electrically longitudinal vibrations travelling along proteins, microtubules and DNA, etc., including interfering with local resonant oscillations by excitation of neighboring molecules and macromolecules [6], for example, C. W. Smith presented a model of ideal cell resonance [7] and Fröhlich presented the coherent oscillation of bio-system in 1978 [8] [9], etc. However, is it possible that this kind of oscillation as a macro-quantum condensation can take place in our body? This problem is interesting because many theories of acupuncture (even including Qigong and Yoga) believe that the down Dan Tian, i.e. about the middle of the abdomen of the human body, is an important accupoint to store bio-energy, until an internal elixir appears [10]. For a long time the internal elixir is an imaginary matter with unclear physical meaning but still pursued by the thousands and thousands practicers, therefore studying this problem is significant for the investigation of mechanism of acupunctures and relative 
points for helping innumerable practicers.

So, in previous works we have presented that the Davydov solitons can condense in two or three potential wells for simulating the oscillation of quasiparticles in the accupoints [11] [12]. In this work, we try to further explore the relative micro-mechanism, namely the condensation through many couple of bio-solitons in a well based on the BCS (Bardeen-Cooper-Schrieffer) superconductor theory extended by the nonlinear operator [13] [14]. Then this model can be used to simulate the creation of internal elixir in the middle of the abdomen of the human body. The meaning of this model is that it provides a mechanism to explain the micro-process of stored bio-energy in our body [15].

\section{Bio-Physical Model}

The nonlinear excitations (solitons) are supposed to have a condensation in a well of the belly. This assumption is based on the Chinese medicine theories and Acupuncture theories, and also from the results of the thousands and thousands practicers of Qigong or Yoga over thousands years of the world [10] [15]. Then from mathematical and physical points of view we can find the conditions: 1) If the interactions between the solitons all have the same frequency of vibration; 2) If they all have the same moment (or opposite moment), then it can be proven that the numbers of solitons which take place interaction are more possible than other cases. So for simplicity, we consider a physical model describe this sort of condensation process, i.e. the two solitons with opposite moments are condensed into a couple of solitons by exchanging an imaginary phonon (or phonon soliton). In this case the Hamiltonian can be written by

$$
H_{\text {int }}=-\frac{1}{2} V \sum_{q, k_{1}, k_{2}} C_{k_{1}+q}^{+} C_{k_{2}-q}^{+} C_{k_{2}} C_{k_{1}},
$$

where $V$ is defined as a coupling number for a couple of solitons, which is constant; $k$ is a wave vector of a soliton,

$$
K=k_{1}+k_{2} \text {, }
$$

$q$ is a wave vector of a (solitonic) phonon, and $C_{k}^{+}\left(C_{k}\right)$ is a creation (annihilation) operator of a soliton with $k$, respectively. Here the quasiparticle is defined as a soliton or a nonlinear excitation generally. So the interaction part of the Hamiltonian can be written as

$$
H_{\text {int }}=-\frac{1}{2} V \sum_{q, k_{1}, k_{2}} C_{k_{1}+q}^{+} C_{K-k_{1}-q}^{+} C_{K-k_{1}} C_{k_{1}} .
$$

Then we can find when $K=0$, the numbers of the solitons participating scattering process is more larger than other situations, hence the above $H_{\text {int }}$ can be written approximately by

$$
H_{\mathrm{int}} \approx-\frac{V}{2} \sum_{k^{\prime}, k} C_{k^{\prime}}^{+} C_{-k^{\prime}}^{+} C_{-k} C_{k},
$$

where $k^{\prime}=k_{1}$.

Therefore, by adding the terms of the energy part for the soliton, the total Hamiltonian can be expressed as 


$$
H=\sum_{k} \varepsilon_{k}\left(C_{k}^{+} C_{k}+C_{-k^{\prime}}^{+} C_{-k}\right)-\frac{V}{2} \sum_{k, k^{\prime}} C_{k^{\prime}}^{+} C_{-k^{\prime}}^{+} C_{-k} C_{k}
$$

\section{Diagonalization of Hamiltonian}

The Hamiltonian $H$ can be firstly simplified by using following forms

$$
\begin{aligned}
& C_{-k} C_{k}=\left\langle C_{-k} C_{k}\right\rangle+\left\{C_{-k} C_{k}-\left\langle C_{-k} C_{k}\right\rangle\right\}, \\
& C_{k}^{+} C_{-k}^{+}=\left\langle C_{k}^{+} C_{-k}^{+}\right\rangle+\left\{C_{k}^{+} C_{-k}^{+}-\left\langle C_{k}^{+} C_{-k}^{+}\right\rangle\right\},
\end{aligned}
$$

and supposing the difference of these operators of the couple of solitons is small value; we therefore can replace the above Equations (6) and (7) into $\left\{C_{k^{\prime}}^{+} C_{-k^{\prime}}^{+} C_{-k} C_{k}\right\}$ and take 1-order of approximation, so the Hamiltonian $H$ is changed as

$$
\begin{aligned}
H= & \sum_{k} \varepsilon_{k}\left(C_{k}^{+} C_{k}+C_{-k}^{+} C_{-k}\right) \\
& -V \sum_{k, k^{\prime}}\left\{C_{k^{\prime}}^{+} C_{-k}^{+}\left\langle C_{-k} C_{k}\right\rangle+C_{-k} C_{k}\left\langle C_{k^{\prime}}^{+} C_{-k^{\prime}}^{+}\right\rangle-\left\langle C_{k^{\prime}}^{+} C_{-k^{\prime}}^{+}\right\rangle\left\langle C_{-k} C_{k}\right\rangle\right\},
\end{aligned}
$$

where we have used

$$
\left\{C_{k}^{+} C_{-k}^{+}-\left\langle C_{k}^{+} C_{-k}^{+}\right\rangle\right\}\left\{C_{-k} C_{k}-\left\langle C_{-k} C_{k}\right\rangle\right\} \approx 0 .
$$

Now, by defining

$$
\begin{aligned}
& \Delta=V \sum_{k}\left\langle C_{-k} C_{k}\right\rangle, \\
& \Delta^{+}=V \sum_{k}\left\langle C_{k}^{+} C_{-k}^{+}\right\rangle,
\end{aligned}
$$

the $H$ can be written as

$$
H=\sum_{k} \varepsilon_{k}\left(C_{k}^{+} C_{k}+C_{-k}^{+} C_{-k}\right)-\Delta \sum_{k}\left(C_{k}^{+} C_{-k}^{+}+C_{-k} C_{k}\right)-\frac{\Delta^{2}}{V},
$$

where notice

$$
\Delta^{2}=\Delta^{+} \Delta
$$

By applying the Heisenberg equation, we get an equation for $C_{k}, C_{-k}$, respectively,

$$
\begin{gathered}
i \frac{\partial C_{k}}{\partial t}=\left[C_{k}, H\right]=\varepsilon_{k} C_{k}-\Delta C_{-k}^{+}, \\
i \frac{\partial C_{-k}}{\partial t}=\left[C_{-k}, H\right]=\varepsilon_{k} C_{-k}+\Delta C_{k}^{+} .
\end{gathered}
$$

For solving these two equations the so-called Bogoliubov transformation is applied,

$$
\begin{aligned}
& \alpha_{k}=u_{k} C_{k}-v_{k} C_{-k}^{+}, \quad \alpha_{k}^{+}=u_{k} C_{k}^{+}-v_{k} C_{-k}, \\
& \alpha_{-k}=u_{k} C_{-k}+v_{k} C_{k}^{+}, \quad \alpha_{-k}^{+}=u_{k} C_{-k}^{+}+v_{k} C_{k},
\end{aligned}
$$

where denote that $\alpha_{k}$ and $\alpha_{-k}$ are also the nonlinear operators due to $C_{k}\left(C_{-k}\right)$ and $C_{k}^{+}\left(C_{k}^{+}\right)$are the nonlinear operators, hence the commutation relations are expressed by the so-called Slash product [13] [14], 


$$
\left[\alpha_{k} / \alpha_{k^{\prime}}^{+}\right]=\left[\alpha_{k} / \alpha_{-k^{\prime}}^{+}\right]=0,
$$

and

$$
\left[\alpha_{k} / \alpha_{k^{\prime}}^{+}\right]=\left[\alpha_{-k} / \alpha_{-k^{\prime}}^{+}\right]=\delta_{k k^{\prime}}\left(u_{k}^{2}+v_{k}^{2}\right)=\delta_{k k^{\prime}} .
$$

For example, for the nonlinear operator $\alpha_{k}^{+}, \alpha_{k}$, we can define a slach product as

$$
\lim _{\lambda \rightarrow 0} \frac{\alpha_{k}^{+}\left(1+\lambda \alpha_{k}\right)-\alpha_{k}^{+}}{\lambda}=\alpha_{k}^{+} / \alpha_{k}+O\left(\lambda^{2}\right) .
$$

Then all properties of the linear operator can be applicable to the $\alpha_{k}^{+} / \alpha_{k}$. Hence by replacing Equations (15) and (16) into the above equations we can get a necessary condition as

$$
u_{k}^{2}+v_{k}^{2}=1 .
$$

So, Equations (15) and (16) can be inverted as

$$
\begin{aligned}
& C_{k}=u_{k} \alpha_{k}+v_{k} \alpha_{-k}^{+}, C_{k}^{+}=u_{k} \alpha_{k}^{+}+v_{k} \alpha_{-k}, \\
& C_{-k}=u_{k} \alpha_{-k}+v_{k} \alpha_{k}^{+}, C_{-k}^{+}=u_{k} \alpha_{-k}^{+}-v_{k} \alpha_{k},
\end{aligned}
$$

by replacing Equations (21) and (22) into the Hamiltonian (11), we get

$$
\begin{aligned}
H \approx & \sum_{k}\left\{\left[\varepsilon_{k}\left(u_{k}^{2}-v_{k}^{2}\right)+2 \Delta u_{k} v_{k}\right]\left(\alpha_{k}^{+} / \alpha_{k}+\alpha_{-k}^{+} / \alpha_{-k}\right)\right. \\
& \left.+\left[2 \varepsilon_{k} u_{k} v_{k}-\Delta\left(u_{k}^{2}-v_{k}^{2}\right)\right]\left(\alpha_{k}^{+} / \alpha_{-k}^{+}-\alpha_{-k} / \alpha_{k}\right)\right\} \\
& +\sum_{k}\left[2 \varepsilon_{k} v_{k}^{2}-2 u_{k} v_{k} \Delta\right]+\frac{\Delta^{2}}{V},
\end{aligned}
$$

where notice again that the Slach product has been introduced. So if let the off-diagnal term in $H$ be equal to zero, we have

$$
\Delta\left(u_{k}^{2}-v_{k}^{2}\right)=2 \varepsilon_{k} u_{k} v_{k},
$$

then by use Equations (20) and (24) we can obtain

$$
\left(u_{k}^{2}\right)^{2}-\left(v_{k}^{2}\right)+\frac{1}{4} \frac{\Delta^{2}}{\xi_{k}^{2}}=0
$$

where

$$
\xi_{k}=\sqrt{\varepsilon_{k}^{2}+\Delta^{2}},
$$

and

$$
\varepsilon_{k}=E_{k}-E_{0} .
$$

Hence, we have

$$
\begin{aligned}
& u_{k}^{2}=\frac{1}{2}\left(1+\frac{\varepsilon_{k}}{\xi_{k}}\right), \\
& v_{k}^{2}=\frac{1}{2}\left(1-\frac{\varepsilon_{k}}{\xi_{k}}\right) .
\end{aligned}
$$

These allow us to obtain the diagnalized Hamiltonian as

$$
H=E_{0}+\sum_{k} \sqrt{\varepsilon_{k}^{2}+\Delta^{2}}\left(\alpha_{k}^{+} / \alpha_{k}+\alpha_{-k}^{+} / \alpha_{-k}\right),
$$


where the energy of basic state is expressed by

$$
E_{s}=2 \sum_{k} \varepsilon_{k} v_{k}^{2}-2 \Delta \sum_{k} u_{k} v_{k}
$$

and the excitation energy is

$$
\xi_{k}=\sqrt{\varepsilon_{k}^{2}+\Delta^{2}},
$$

so $\Delta$ is a energy gap for realizing the condensation, and $\varepsilon_{k}$ is the energy of a soliton with the wave vector $k$.

\section{The Calculation of $\Delta(t)$}

When the system arrives at the lowest condensation temperature $T_{c}$ we must have the number of the couple of solitons is zero, so

$$
\left\langle\alpha_{-k} \alpha_{k}\right\rangle_{T_{c}}=\left\langle\alpha_{k}^{+} \alpha_{-k}^{+}\right\rangle_{T_{c}}=0,
$$

then we get

$$
\begin{aligned}
\Delta & =V \sum_{k}\left\langle C_{-k} C_{k}\right\rangle_{T_{c}} \\
& =V \sum_{k}\left\langle\left(u_{k} \alpha_{-k}+v_{k} \alpha_{k}^{+}\right)\left(u_{k} \alpha_{k}+v_{k} \alpha_{-k}^{+}\right)\right\rangle_{T_{c}} \\
& \approx V \sum_{k} u_{k} v_{k}\left(\left\langle\alpha_{-k} / \alpha_{-k}^{+}\right\rangle_{T_{c}}-\left\langle\alpha_{k}^{+} / \alpha_{k}^{+}\right\rangle_{T_{c}}\right) \\
& =V \sum_{k} u_{k} v_{k}\left(1-\left\langle\alpha_{k}^{+} / \alpha_{k}^{+}\right\rangle_{T_{c}}-\left\langle\alpha_{-k}^{+} / \alpha_{-k}\right\rangle_{T_{c}}\right),
\end{aligned}
$$

and

$$
\begin{aligned}
\left\langle\alpha_{k}^{+} / \alpha_{k}^{+}\right\rangle_{T_{c}} & =\left\langle\alpha_{-k}^{+} / \alpha_{-k}\right\rangle_{T_{c}} \approx f\left(\xi_{k}\right)= \\
& =\left[\exp \left(\frac{\xi_{k}}{k_{B} T_{c}}\right)-1\right]^{-1},
\end{aligned}
$$

where denote

$$
\Delta\left(T_{c}\right)=V \sum_{k} u_{k} v_{k}\left(1-2 f\left(\xi_{k}\right)\right) .
$$

By considering Equations (28) and (29) replaced into Equation (36) to cancel $\Delta(T)$, we get

$$
\begin{aligned}
1 & =\frac{1}{2} V \sum_{k} \frac{1-2 f\left(\xi_{k}\right)}{\xi_{k}} \\
& =\frac{1}{2} V \sum_{k} \frac{\tanh \left(\frac{1}{2 k_{B} T} \xi_{k}\right)}{\xi_{k}},
\end{aligned}
$$

finally we can get

$$
\Delta(T)=\pi k_{B} T_{c} \sqrt{\frac{8}{7 \varsigma(3)}}\left(1-\frac{T}{T_{c}}\right)^{\frac{1}{2}}, \text { for } T_{c}-T \ll T_{c},
$$

and 


$$
k_{B} T_{c}=\frac{2 e^{\gamma}}{\pi} \omega \exp \left[-\frac{1}{g(T) V}\right] \text {, }
$$

where $\gamma$ is the Eular number, about 0.5772 , so $\frac{2 e^{\gamma}}{\pi}$ is about 1.13. Normally for bio-solitons condensation in our body the temperature $T_{c}$ should make sense at room temperature, namely

$$
T_{c} \approx \frac{1.13}{k_{B}} \omega \exp \left[-\frac{1}{g\left(T_{c}\right) V}\right] \approx 300(K),
$$

where the bio-system can regulate the $\omega, g\left(T_{c}\right)$ and $V$ automatically to enable $T_{c}$ in the interval of room temperature. On the other hand, since $T_{c}-T \ll T_{c}, T_{c}>T$ so, when $T$ increases, $\Delta(T)$ decreases; this means when temperature increase the realization of the couple of bio-solitons is easier than temperature decrease. Moreover, because the momentum of the couple of solitons are invariant, therefore the velocities of their movements are invariant without resistance from the medium.

\section{Big Soliton}

Furthermore if we assume that $\Delta$ is complex number described by

$$
\Delta=V \sum_{k}\left\langle C_{-k} C_{k}\right\rangle=|\Delta| e^{i \theta},
$$

then $u_{k}$, and $v_{k}$ are also complex. Then using the same approach as the above we can get

$$
\frac{v_{k}}{u_{k}}=\Delta \frac{\xi_{k}-\varepsilon_{k}}{|\Delta|^{2}}
$$

then since $\xi_{k}-\varepsilon_{k}$ and $|\Delta|^{2}$ are all real numbers, so the complex numbers $\frac{v_{k}}{u_{k}}$ and $\Delta$ have the same phase $\theta$. Then from the spectral decomposition of the diagonalized Hamiltonian (30) we can find that the wave functions of the couple of solitons also share the same phase $\theta$, therefore a highly ordered couple of solitons can be supposed by using a wave function to describe

$$
\phi(r, t)=\varphi(r, t) \phi_{0} e^{j \theta(r, t)},
$$

where defining

$$
\phi_{0}^{2}=\frac{\alpha}{2 \lambda}
$$

Then in the situation of no external field condition, which is just a suitable condition for the oscillation inside of the belly, we have the density of the free energy expressed as

$$
\begin{aligned}
f_{s} & =f_{0}-\frac{1}{2 m^{2}}|\nabla \phi|^{2}-\alpha|\phi|^{2}+\lambda|\phi|^{4}+\cdots \\
& \approx f_{0}-\frac{1}{2 m^{2}}|\nabla \phi|^{2}-\alpha|\phi|^{2}+\lambda|\phi|^{4}
\end{aligned}
$$

then applying the principle of minium of the free energy we get 


$$
\delta \int f \mathrm{~d}^{3} x=0
$$

which allows us to have

$$
\frac{1}{2 m^{2}} \nabla^{2} \phi-\alpha \phi+2 \lambda \phi^{3}=0
$$

This is just a kind of Ginzburg and Landau equation which can describe a soliton. For example in the three dimensional case we can have

$$
\frac{1}{2 m} \frac{\partial^{2}}{\partial Y^{2}} \phi+\eta \phi-2 \lambda \phi^{3}=0
$$

where defining

$$
Y=a_{1} x+a_{2} y+a_{3} z
$$

Then the solution of Equation (48) can be gotten by

$$
\phi= \pm \sqrt{\frac{\eta}{2 \lambda}} \sec h\left[\frac{a_{1}\left(x-x_{0}\right)+a_{2}\left(y-y_{0}\right)+a_{3}\left(z-z_{0}\right)}{\sqrt{2} \xi(T)\left(a_{1}^{2}+a_{2}^{2}+a_{3}^{2}\right)}\right],
$$

where notice $\xi(T)$ is defined as the coherent length, namely

$$
\xi(T)=\frac{1}{2 m|\eta|} \text {. }
$$

This $\phi$ is just a soliton! This shows that the gathering of the couple of solitons in the well can form a big soliton, which means the condensation of the many solitons finally can form a big bio-soliton with the oscillation in the well of bio-system.

\section{Conclusion}

A couple of bio-solitons condensation has been presented. The two solitons with opposite moments are condensed into a couple of solitons by exchanging imaginary phonons. Then these couple of solitons can form a big soliton with oscillation. The process can be taken place in the room temperature, and the higher temperature makes the realization easier than lower temperature. This model can be used to simulate the bio-energy stored in our body such as the middle of abdomen of the human body, and also provides a kind explanation of mechanism for the internal elixir formation. This means that the so called internal elixir may be formed through the macro-quantum condensation of a couple of solions, and then the condensation plays a sort of oscillation as a big (or macro-) soliton.

\section{References}

[1] Davydov, A.S. (1979) Physica Scripta, 20, 387-396. https://doi.org/10.1088/0031-8949/20/3-4/013

[2] Davydov, A.S. and Kislukha, N.I. (1973) Physica Status Solidi (b), 59, 465-470. https://doi.org/10.1002/pssb.2220590212

[3] Davydov, A.S. (1973) Journal of Theoretical Biology, 38, 559-569. https://doi.org/10.1016/0022-5193(73)90256-7 
[4] Pang, X.F. and Feng, Y.-P. (2005) Quantum Mechanics in Nonlinear Systems. World Scientific Publishing Co. Pte. Ltd., Singapore City. https://doi.org/10.1142/5721

[5] Pang, X.-F. (2000) Physical Review E, 62, 6989-6998. https://doi.org/10.1103/PhysRevE.62.6516

[6] Geesink J.H. and Meijer D.K.F. (2016) Bio-Soliton Model That Predicts NonThermal Electromagnetic Radiation Frequency Bands, That Either Stabilize or Destabilize Life Conditions. Bio. phys., arXiv:1610.04855.

[7] Smith, C.W. and Best, S. (1989) Electromagnetic Man. J. M. Dent Ltd., London.

[8] Fröhlich, H. (1988) Biological Coherence and Response to External Stimuli. Springer-Vering, Berlin. https://doi.org/10.1007/978-3-642-73309-3

[9] Fröhlich, H. (1982) Coherent Excitation in Biological System. Springer-Vering, Berlin.

[10] Needham, J. (2015) Science and Civilisation in China. Vol. 5, Part 5, Chemistry and Chemical Technology, Cambridge University Press, London, New York.

[11] Bi, Q. (2016) Information Soliton and Excitation from Nonlinearity. LAP LAMBERT Academic Publishing, Germany.

[12] Bi, Q. (2016) Journal of Modern Physics, 7, 1811-1817. https://doi.org/10.4236/jmp.2016.713161

[13] Schwartz, C. (1997) Journal of Mathematical Physics, 38, 484-500. https://doi.org/10.1063/1.531829

[14] Schwartz, C. (1997) Journal of Mathematical Physics, 38, 38-41. https://doi.org/10.1063/1.532070

[15] Needham, J. (2011) Science and Civilisation in China. Vol. 5, Part 2, Chemistry and Chemical Technology, Cambridge University Press, London, New York.

Submit or recommend next manuscript to SCIRP and we will provide best service for you:

Accepting pre-submission inquiries through Email, Facebook, LinkedIn, Twitter, etc. A wide selection of journals (inclusive of 9 subjects, more than 200 journals) Providing 24-hour high-quality service User-friendly online submission system Fair and swift peer-review system Efficient typesetting and proofreading procedure Display of the result of downloads and visits, as well as the number of cited articles Maximum dissemination of your research work

Submit your manuscript at: http://papersubmission.scirp.org/

Or contact jmp@scirp.org 